\title{
Nonintegrable interaction of ion acoustic and electromagnetic waves in a plasma
}

\author{
F. B. Rizzato, ${ }^{1}$ S. R. Lopes, ${ }^{2}$ and A. C.-L. Chian ${ }^{3}$ \\ ${ }^{1}$ Instituto de Física, Universidade Federal do Rio Grande do Sul, P.O. Box 15051, 91501-970 Porto Alegre, \\ Rio Grande do Sul, Brazil \\ ${ }^{2}$ Departamento de Física, Universidade Federal do Paraná, P.O. Box 19081, 81531-990 Curitiba, Paraná, Brazil \\ ${ }^{3}$ National Institute for Space Research-INPE, Post Office Box 515, 12227-010 São José dos Campos, São Paulo, Brazil
}

(Received 31 October 1996)

\begin{abstract}
In this paper we reexamine the one-dimensional interaction of electromagnetic and ion acoustic waves in a plasma. Our model is similar to one solved by Rao et al. [Phys. Fluids 26, 2488 (1983)] under a number of analytical approximations. Here we perform a numerical investigation to examine the stability of the model. We find that for slightly overdense plasmas, the propagation of stable solitary modes can occur in an adiabatic regime where the ion acoustic electric-field potential is enslaved to the electromagnetic field of a laser. However, if the laser intensity or plasma density increases or the laser frequency decreases, the adiabatic regime loses stability via a transition to chaos. Different asymptotic states are attained when the adiabatic regime no longer exists. In these states the plasma becomes rarefied and the laser field tends to behave like a vacuum field. [S1063-651X(97)05603-1]
\end{abstract}

PACS number(s): 52.35.Mw, 05.45.+b

\section{INTRODUCTION}

The recent developments of laser technologies allow the creation of strong pulses that can propagate in a plasma, either to accelerate particles [1] or to induce laser-assisted fusion [2]. Now, if a laser pulse interacts with a plasma, the possibility exists of nonlinear wave coupling involving the pulse itself and nonlinear plasma modes [2,3]. Since the variety of these nonlinear plasma modes is large and since each mode exhibits a considerable richness regarding amplitude, polarization, and frequency range [4,5], the nonlinear mode coupling is a feature to be appreciated with care.

One important example of nonlinear mode coupling concerns the interaction of relativistically strong electromagnetic waves with Langmuir waves [6-8]. These analyses are restricted to simple wave solutions with well-defined propagation velocities that are superluminous in an unmagnetized plasma. For these superluminous waves it is shown that the wave dynamics can be viewed as similar to the coupled dynamics of two nonlinear oscillators with their natural frequencies given by real numbers. One of the oscillators describes the transverse electromagnetic field and the other the longitudinal electrostatic field. The dynamics is found to be predominantly integrable. Some nonlinear resonant island chains are present in the appropriate Poincaré maps [8], but their overlap is so small that the resulting trajectories are mostly regular.

In another important range of subluminous wave velocities, around the ion acoustic range, the laser wave can couple to ion acoustic plasma modes. In this case the coupled waves propagate with velocities close to the ion acoustic velocity, which is much smaller than the velocity of light. Here the ionic dynamics plays a crucial role and the resulting dynamics, in principle, bears no resemblance to the dynamics described in the preceding paragraph. A good deal of analytical work has been done in Ref. [9] to show that if one assumes once more a common and constant propagation velocity, the system becomes again equivalent to a pair of nonlinearly coupled oscillators. A different feature studied here is that when the laser frequency is smaller than the average plasma frequency, wave localization can occur such that one has the formation of intense solitary pulses. In terms of the system of equivalent oscillators, the possibility of solitary pulses occurs when by varying the appropriate parameters the linear frequency of that oscillator describing the laser wave becomes imaginary. Using the language of nonlinear dynamics, this change in the character of the frequency occurs because the central elliptic point present in the appropriate phase space turns into a hyperbolic point [10].

The work by Rao et al. [9] utilizes powerful approximation techniques, but is essentially analytical as commented before. Therefore, one would like to have some information on the stability of the solitary pulses thus formed, and this is what we do here. The stability issue has already been addressed several times over the past years, but the focus has been preferentially directed upon the linear perspective [11]. However, if one wishes to have some additional insight into the nonlinear development of these instabilities, the most appropriate tools of investigation appear to be the Poincare maps mentioned above. With these maps one records the phase-space coordinates of one of the oscillators, as one of the coordinates of the other crosses its zero with a definite sign for the derivative. Since the system is two degrees of freedom and since there exists a conserved Hamiltonian for the system, the point recorded on the map gives all the relevant information for the dynamics. Regular motion is associated with smooth curves of the Poincare maps and chaotic (or nonintegrable) motion is associated with an erratic distribution of points representing the trajectory. We find here that the stability of the solitary pulses is quite limited. In fact, we found no regular motion for velocities sufficiently below the ion acoustic velocities and for effective laser frequencies sufficiently below the average plasma frequency. In those chaotic situations the final asymptotic behavior looks like the one corresponding to uncoupled waves in the vacuum. The intrinsic instability of the system for too low values of the 
effective laser wave frequency, in particular, seems to preclude the formation of some special soliton solutions, as we shall see. Chaos is present because our system is two degrees of freedom and the system is two degrees of freedom because we allow for charge separation in the ion acoustic dynamics. Had we used quasineutrality assumptions, the system would be one degree of freedom and chaotic solutions would be absent. We finally point out that the transition to chaos we detect here is of a conservative character, so the chaotic dynamics we expect to see is of different type from the one present in dissipative systems $[5,12]$.

The paper is organized as follows. In Sec. II we review the basic theory governing the interaction of a laser and an ion acoustic mode. In Sec. III we analyze the nonintegrable dynamics with help of Poincaré maps. In Sec. IV we conclude the work.

\section{BASIC THEORY}

\section{A. Introductory remarks}

We consider here the interaction of a laser wave and an ion acoustic mode in a globally neutral plasma consisting of light electrons and massive ions. Let us rederive the governing equation for the laser mode in a slightly different way from the one used in Ref. [9]. If one assumes that the waves are plane waves propagating along the $z$ axis, the equation governing the high-frequency laser dynamics can be written in the form

$$
\frac{\partial^{2} \Psi}{\partial t^{2}}-c^{2} \frac{\partial^{2} \Psi}{\partial z^{2}}=-\frac{4 \pi q^{2}}{m_{e}}\left(n_{0}+\delta n_{e}\right) \frac{\Psi}{\sqrt{1+|\Psi|^{2}}},
$$

where $-q$ is the electron charge, $m_{e}$ its rest mass, $n_{0}$ the average density, $\delta n_{e}$ the fluctuations of the electronic density due to the action of the waves, and $c$ the velocity of light. In Eq. (1) the laser intensity is considered strong enough to drive electrons to relativistic velocities. The field $\Psi(z, t)$ is defined in terms of the relation

$$
\frac{q \mathbf{A}}{m_{e} c^{2}}=\frac{1}{2}(\hat{\mathbf{x}}-i \hat{\mathbf{y}}) \Psi+\text { c.c. }
$$

where $\mathbf{A}$ is the vector potential of a circularly polarized laser field, with $i^{2}=-1$ and c.c. designating complex conjugate. Now we assume solutions of the form

$$
\Psi(z, t)=\psi(\xi) e^{i(k z-\omega t)},
$$

with $k$ and $\omega$, respectively the wave vector and the effective frequency of the laser, with $\xi \equiv z-V t$ and $\psi$ a real slowly varying variable; $V$ denotes the propagation velocity. Next we substitute relation (3) into Eq. (1) and separate the resulting equation into its real and imaginary parts. The imaginary component yields the relation

$$
V=\frac{c^{2} k}{\omega}
$$

from which one can determine the propagation velocity, given the frequency and wave vector of the carrier. The real part, on the other hand, yields a governing equation for the real amplitude $\psi$, valid when $V \ll c$,

$$
\beta \frac{d^{2} \psi}{d \xi^{2}}=-\Delta \psi+\left(1+\delta n_{e}\right) \frac{\psi}{\sqrt{1+\psi^{2}}} .
$$

In Eq. (5), space is normalized by the electronic Debye length $\lambda_{\mathrm{De}_{2}}=\left(T_{e} / 4 \pi n_{0} q^{2}\right)^{1 / 2}$, time by the ion plasma frequency $\omega_{p i}^{2}=4 \pi n_{0} q^{2} / m_{i}$, and density by $n_{0} ; m_{i}$ is the ion mass, $T_{e}$ is the electron temperature, $\beta=c^{2} / v_{t e}^{2} \gg 1$, $\Delta=\omega^{2} / \omega_{p e}^{2}, v_{t e}^{2}=T_{e} / m_{e}$, and $\omega_{p e}^{2}=4 \pi n_{0} q^{2} / m_{e}$. Note that in our calculations the character of the electromagnetic wave is determined by whether $\Delta$ is larger or smaller than unity. In the former case one has propagation in an underdense plasma and in the latter case one has propagation in an overdense plasma. Since it can be seen in Eq. (5) that the coefficient of the linear $\psi$ term is given by $1-\Delta$, wave localization in overdense situations occurs when the natural frequency of the associated oscillator can be interpreted as an imaginary number. Our factor $\Delta$ is defined in terms of the effective frequency $\omega$, which incorporates all possible frequency shifts. Therefore, this factor $\Delta$ replaces, in a simplified way, the slightly more complicated total frequency shift introduced in Ref. [9]. This is why we adopt the present formalism.

As for the ion acoustic field excited by ponderomotive effects associated with the electromagnetic wave, we simply write down the governing equation obtained from the analysis of the low-frequency dynamics involving the ion acoustic electric-field potential $\Phi$, the massless warm electronic fluid, and the massive cold ionic fluid. One has [9]

$$
\frac{d^{2} \Phi}{d \xi^{2}}=-\frac{M}{\sqrt{M^{2}-2 \Phi}}+e^{\Phi+\beta-\beta \sqrt{1+\psi^{2}}}
$$

where $\Phi$ has been normalized by $T_{e} / q$ ( $q$ is the ion charge) and we have introduced the Mach number $M=V / C_{s}$ with the ion acoustic velocity $C_{s}$ written in the form $C_{s}$ $=\left(T_{e} / m_{i}\right)^{1 / 2}$. The ions are considered nonrelativistic due to their large mass. We point out here that the total ion and electron densities are respectively measured by the absolute values of the first and second terms on the right-hand side of Eq. (6); in particular, the fluctuating electron density obtained under the assumption of massless electrons,

$$
\delta n_{e}=e^{\Phi+\beta-\beta \sqrt{1+\psi^{2}}}-1,
$$

is the expression to be used in Eq. (5).

Equations (5)-(7) govern the nonlinearly coupled dynamics of the dynamical variables $\psi(\xi)$ and $\Phi(\xi)$. We assume propagation at a constant velocity $M$ and take into consideration full nonlinear effects in both the ion acoustic and the transverse relativistic dynamics of electrons. It is worth mentioning that alternative approaches do not restrict the spacetime dependence of the solutions, but use weak nonlinear expansions instead [13-15].

Now, as shown in the paper by Rao et al. [9], Eqs. (5) and (6) can be obtained from a generalized two degrees of freedom Hamiltonian $H$, 


$$
H=\frac{P_{\psi}^{2}}{2 \beta^{2}}-\frac{P_{\Phi}^{2}}{2}-\frac{\Delta \beta}{2} \psi^{2}+M \sqrt{M^{2}-2 \Phi}+e^{\Phi+\beta-\beta \sqrt{1+\psi^{2}}},
$$

where a misprint in [9] has been corrected. In the Hamiltonian, $P_{\psi}$ and $P_{\Phi}$ are the canonical momenta conjugate to the corresponding subscript coordinates. Hence our system contains four dimensions: $\psi, P_{\psi}, \Phi$, and $P_{\Phi}$.

Since the Hamiltonian (8) does not depend explicitly on the "time" coordinate $\xi$, it is a constant of motion. We will be interested in finding solitary waves and all those solutions towards which initial solitary waves can evolve in time if they are unstable. Therefore, we shall work on the particular energy surface that allows for the presence of the configuration $\Phi=\psi=P_{\Phi}=P_{\psi}=0$, since this configuration is in fact the appropriate asymptotic solution for solitary pulses. We recall that from the canonical equations generated by $H$, Eq. (8), it follows that $d \Phi / d \xi=-P_{\Phi}, d \psi / d \xi=P_{\psi} / \beta^{2}$, and the boundary conditions for a solitary pulse are, in the more traditional form, $\Phi, \psi, d \Phi / d \xi$, and $d \psi / d \xi \rightarrow 0$ as $|\xi| \rightarrow \infty$. The above considerations enable one to determine the constant numerical value of the Hamiltonian; it reads $H=1+M^{2}$.

The Hamiltonian supports pure ion acoustic waves; it is easy to see that regardless of the values of $P_{\Phi}$ and $\Phi$, if $\psi(\xi=0)=P_{\psi}(\xi=0)=0$, then $\psi(\xi)=P_{\psi}(\xi)=0$ at any $\xi$. This is not true for electromagnetic waves. Even if one starts with a laser pulse in the absence of any space-charge fields $\left(\Phi=P_{\Phi}=0\right)$, Eq. (6) indicates that the dynamics of the electric-field potential is subsequently driven by a $\psi^{2}$ factor.

We shall restrict the present analysis to subsonic cases, where $M<1$. This causes the coefficient of the linear $\Phi$ term in Eq. (6) to assume only negative values. As for the laser field, we focus attention on overdense plasmas with $\Delta<1$. Since the linear coefficient of Eq. (5) is given by a factor $(1-\Delta) / \beta$, as already mentioned, the system is linearly unstable against the generation and propagation of electromagnetic modes. Nonlinear saturation of the unstable process may be responsible for the creation of solitary pulses.

\section{B. Adiabatic approximation}

It has been seen that laser localization occurs in overdense plasmas where $\Delta<1$. Since, in general, $\Delta$ is only slightly different from unity and since the small factor $1-\Delta$ is yet to be divided by the large factor $\beta$ to obtain the coefficient of the linear term of Eq. (5), the conclusion is that, in general, the following relation may hold:

$$
\left(\frac{1-\Delta}{\beta}\right) \ll\left|1-\frac{1}{M^{2}}\right| \text {. }
$$

But if such a relation does hold, it is likely that the dynamics on the $\left(\psi, P_{\psi}\right)$ phase plane tends to be much slower than the corresponding dynamics developing on the $\left(\Phi, P_{\Phi}\right)$ phase plane. In this limiting case one could be tempted to use the results of the center manifold and adiabatic theorems [16], which say that the integration on the latter phase plane could be done simply by taking Eq. (6) with $\psi^{2}$ considered as a constant factor. In addition, as $\psi$ slowly evolves as a func-

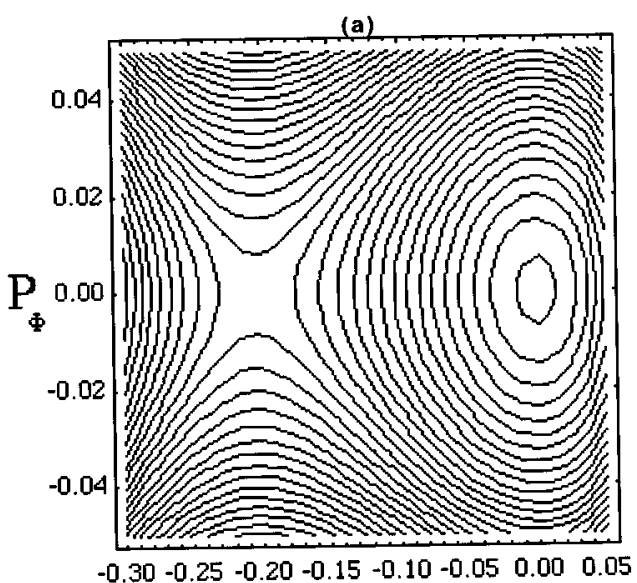

$\Phi$

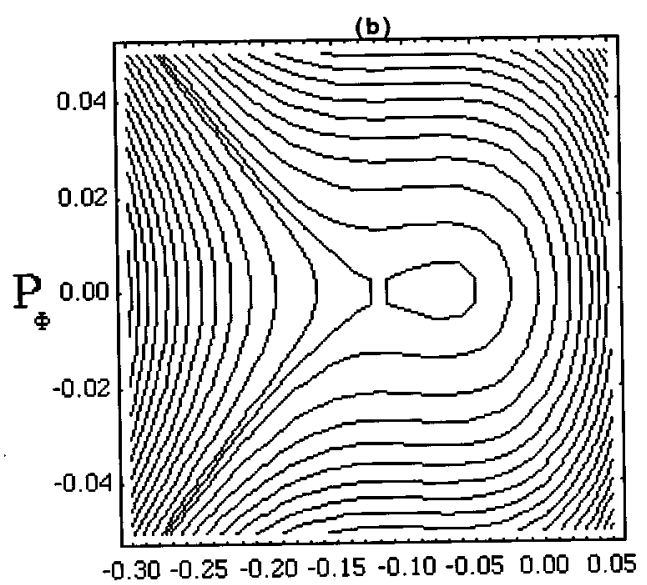

$\Phi$

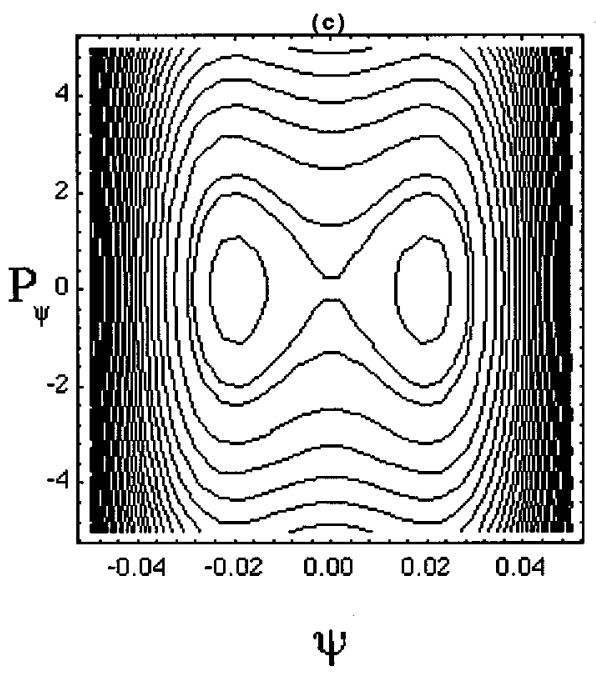

FIG. 1. Phase space on the $\left(\Phi, P_{\Phi}\right)$ plane for fixed values of $\psi$, with $M=0.9$ and $\beta=100$ : (a) $\psi=0$ and (b) $\psi=0.001$. (c) $\left(\psi, P_{\psi}\right)$ phase space using $\Delta=0.98$.

tion of $\xi$, the $\Phi$ dynamics evolves in such a way as to conserve the action integral $(1 / 2 \pi) \oint P_{\Phi} d \Phi$. In Fig. 1 we take fixed values of $\psi$ to plot contour levels of the driven ion acoustic Hamiltonian, which is here defined as 


$$
H_{\Phi}=-\frac{P_{\Phi}^{2}}{2}+M \sqrt{M^{2}-2 \Phi}+e^{\Phi+\beta-\beta \sqrt{1+\psi^{2}}} .
$$

In the adiabatic regime this is the Hamiltonian controlling the fast motion on the $\left(\Phi, P_{\Phi}\right)$ plane. For vanishingly small values of $\psi^{2}$ one can see in Fig. 1(a) an elliptic fixed point at the origin and a hyperbolic point located at $\Phi<0$; trajectories move counterclockwise and the typical parameters $\beta=100$ and $M=0.9$ are considered. Now as $\psi^{2}$ grows the elliptic point moves toward the hyperbolic point, as seen in Fig. 1(b). For large enough values of $\psi$ the elliptic and hyperbolic fixed points coalesce in an inverse saddle-node bifurcation [17]; we shall refer to this coalescence as collapse. For completeness we display in Fig. 1(c) the $\left(\psi, P_{\psi}\right)$ phase space obtained from the full Hamiltonian (8) when we set $\Phi \rightarrow 0$ and $P_{\Phi} \rightarrow 0$. Note that when $\Delta<1$, as in Fig. 1(c), where we consider $\Delta=0.98$, the fixed point at the origin is hyperbolic; for $\Delta>1$ the fixed point would be elliptic.

A delicate point about the adiabatic approach is that the adiabatic theory may be expected to work relatively well only if the maximum value of $\psi^{2}$ throughout the entire dynamics is much smaller than the critical value for which the collapse does occur. Indeed, if this is the case, the rotational frequency around the elliptic point on the $\left(\Phi, P_{\Phi}\right)$ plane can be expected to be larger enough than the characteristic time scale of the $\psi$ dynamics. But, on the other hand, if the maximum value of $\psi^{2}$ becomes too close to the critical value, the rotational frequency tends to diminish and attain values comparable to the $\psi$ time scales. The rotational frequency actually vanishes at the collapse. From this point of view, Eq. (9) may not be sufficient to guarantee adiabaticity since it is derived on the basis of linearization procedures, where the fields are assumed to be much smaller than the maximum values they can actually attain as time elapses. With that in mind we now proceed to derive a validity condition for adiabaticity that takes into account the nonlinear effects associated with the finiteness of $\psi^{2}$. We shall see that the adiabatic range is in fact much smaller than the one suggested by Eq. (9).

Let us then examine the adiabatic trajectory of the elliptic point on the $\left(\Phi, P_{\Phi}\right)$ plane. Our interest lies in the fact that the existence condition for this point provides a reasonably good estimate of the range of validity of the adiabatic regime; we emphasize that adiabaticity is expected to break down when the elliptic and hyperbolic points cease to exist. One can use the results from the center manifold theorem to estimate the location of slowly moving fixed points,

$$
\left.\frac{\partial H_{\Phi}}{\partial \Phi}\right|_{\text {fixed }}=\left.\frac{\partial H_{\Phi}}{\partial P_{\Phi}}\right|_{\text {fixed }}=0
$$

For a given value of $\psi$, we thus have

$$
e^{\Phi_{\text {fixed }}+\beta-\beta \sqrt{1+\psi^{2}}}-\frac{M}{\sqrt{M^{2}-2 \Phi_{\text {fixed }}}}=0
$$

from which a series expansion yields a relation correct up to quadratic terms

$$
\psi^{2}=\frac{2}{\beta}\left(1-\frac{1}{M^{2}}\right) \Phi_{\text {fixed }}+\frac{1-2 \beta-2 M^{2}+M^{4}}{\beta^{2} M^{4}} \Phi_{\text {fixed }}^{2}
$$

as for $P_{\Phi}$ one has $P_{\Phi, \text { fixed }}=0$. We point out that in the extreme adiabatic limit where frequency shifts are vanishingly small, $\Delta \rightarrow 1$, the first term on the right-hand side of Eq. (13) coincides with expression (35) of Ref. [9].

Given $\psi$, the quadratic relation above furnishes two roots in the variable $\Phi$ if the appropriate discriminant is positive. One of the roots represents the elliptic point, which we shall call $\Phi_{\text {ell }}\left(\psi^{2}\right)$, and the other represents the hyperbolic point $\Phi_{\text {hyp }}\left(\psi^{2}\right)$, both seen in Figs. 1(a) and 1(b). What must be done now is to substitute the adiabatic relation $\Phi_{\text {ell }}=\Phi_{\text {ell }}\left(\psi^{2}\right)$ into Eq. (5) to determine and examine the slow dynamics on the $\left(\psi, P_{\psi}\right)$ phase plane. Assuming for a moment $|\Phi|$ and $\psi^{2}$ small, which shall be seen to be true if $\Delta$ is sufficiently close to unity, we drop the quadratic $\Phi$ term in Eq. (13) and obtain

$$
\Phi_{\mathrm{ell}} \approx \frac{1}{2} \frac{M^{2} \beta}{M^{2}-1} \psi^{2},
$$

which shows that $\Phi<0$ if $M<1$. Therefore, in the present approximation the $\psi$ dynamics is commanded by the effective potential written, apart from a global multiplicative constant, as

$$
V_{\mathrm{eff}}(\psi)=\frac{1}{2}(\Delta-1) \psi^{2}+\frac{1}{8}\left(1+\beta-\frac{M^{2} \beta}{M^{2}-1}\right) \psi^{4} .
$$

It is thus seen that near the ion acoustic resonance where $M \approx 1$, the electric-field potential response satisfies $|\Phi| \gg \psi^{2}$ and essentially determines the adiabatically saturated value of the laser field

$$
\psi_{\max }^{2} \approx 4 \frac{(\Delta-1)\left(M^{2}-1\right)}{\beta} .
$$

The approximation we have used in Eqs. (15) and (16) assumes that the quadratic $\Phi$ term in relation (13) is much smaller than the others. This is true only if one is sufficiently away from that situation where the discriminant vanishes causing the collapsing of elliptic and hyperbolic points. Near the collapse, in particular, the adiabatic approximation is expected to break down. Given $M$ and $\beta$, use of Eq. (16) and the self-consistency requirement of a non-negative discriminant for relation (13) finally yields a complicated relation that can be used as an estimate for the critical value of $\Delta, \Delta_{\mathrm{cr}}$, below which approximation (14) is no longer valid,

$$
\Delta<\Delta_{\mathrm{cr}} \equiv \frac{-\left(-4+7 \beta+8 M^{2}+\beta M^{2}-4 M^{4}\right)}{4\left(1-2 \beta-2 M^{2}+M^{4}\right)} \approx \frac{7+M^{2}}{8} .
$$

As $\Delta$ starts to get too close to $\Delta_{\text {cr }}$ the adiabatic approximations are expected to get poorer and poorer. Condition (17) is a rough estimate that could be refined with more detailed algebraic work. However, we shall take it as sufficiently accurate and complete for our purposes. In any case, the ultimate answer is yet to be given by numerical work, as we will do next. 


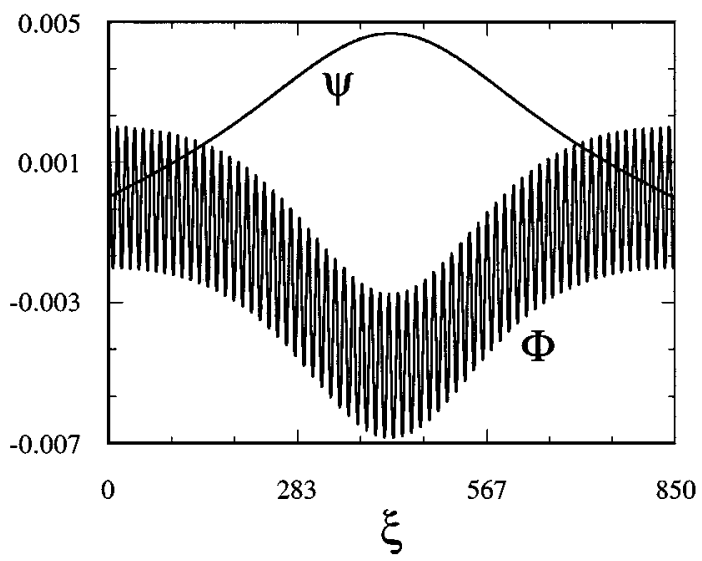

FIG. 2. Time series in the adiabatic regime: $M=0.9, \beta=100$, and $\Delta=0.99$.

\section{TRANSITION FROM ADIABATIC TO CHAOTIC REGIMES}

\section{A. Testing adiabaticity}

In all the following numerical applications we use $\beta=100$ and $M=0.9$, which yield $\Delta_{\mathrm{cr}} \approx 0.976$. We promptly conclude that the validity range for the adiabatic regime is quite narrow, as a matter of fact, much narrower than the range predicted by Eq. (9). Indeed, for the chosen values of $\beta$ and $M$ and considering overdense plasmas, Eq. (9) basically imposes no essential restriction on the value of $\Delta$, a failure of the linear theory as mentioned before. In the following we shall see that the estimate based on $\Delta_{\mathrm{cr}}$, Eq. (17), is much more accurate than the one based on Eq. (9) and that the destruction of the adiabatic regime is in fact associated with a transition to chaos.

Before examining the validity ranges and the transition, let us first perform some initial simulations of Eqs. (5) and (6) to make sure that the adiabatic regime is in fact present if condition (17) is safely observed. To do so we start a single initial condition with $\Delta=0.99$, and with $\psi=P_{\Phi}=0$, $\Phi=0.0005$, and $P_{\psi}=0.0024$ such that $H=1+M^{2}$. We plot the time series for $\psi(\xi)$ and $\Phi(\xi)$ in Fig. 2. In the figure we see that $\Phi$ undergoes a fast oscillatory motion, while $\psi$ evolves in a much slower time scale. The adiabatic features can be visualized also on the $\left(\psi, P_{\psi}\right)$ phase plane as in Fig. 3 . In the figure we compare three solitary trajectories: the exact trajectory, the adiabatic trajectory calculated from Eq. (5) under the assumption (14), and the trajectory calculated from Eq. (5) under the assumption $\Phi \rightarrow 0$. The adiabatic trajectory yields a fairly good approximation to the actual trajectory. Here we use $\Delta=0.98$. This chosen value of $\Delta$ is slightly smaller than in Fig. 2 because it allows a clearer view of the differences between adiabatic and exact trajectories. For values of $\Delta$ closer to unity the approximation gets better and better until such a point where no distinction can be appreciated.

\section{B. Transition}

Now the question is what happens as the parameters are varied beyond the validity range for the adiabatic regime. To simplify the discussion we shall focus attention on the behavior of the system as $\Delta$ decreases. As our system is Hamil-

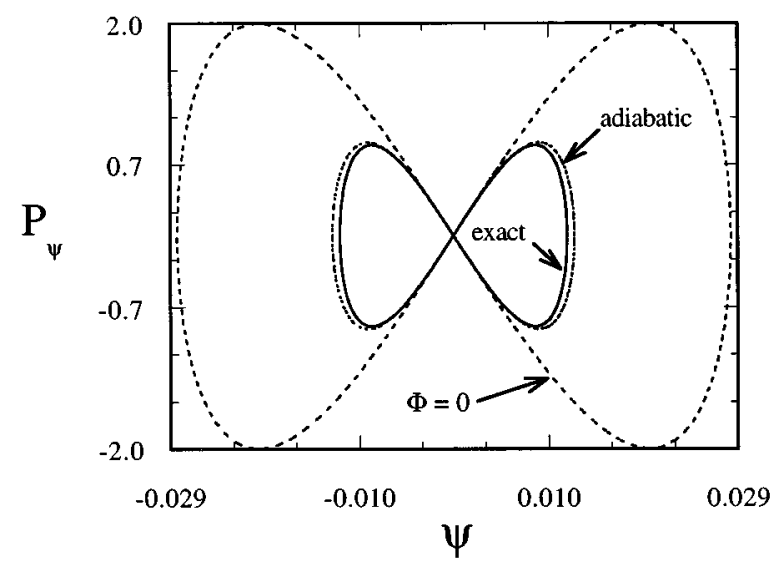

FIG. 3. Accuracy of the adiabatic approximation tested on the $\left(\psi, P_{\psi}\right)$ plane: $M=0.9, \beta=100$, and $\Delta=0.98$.

tonian [see Eq. (8)] with two degrees of freedom, we make use of the Poincare map methodology and plot the pair of phase variables $\Phi$ and $P_{\Phi}$ each time $P_{\psi}=0$ with $d P_{\psi} / d \xi>0$. Several initial conditions are launched with the numerical values for $\Phi(\xi=0)$ distributed within a small range typically satisfying $-0.001<\Phi(\xi=0)<0.001$. Similarly to the initial simulation presented before, for all initial conditions we always take $\psi=P_{\Phi}=0$ and calculate the corresponding initial $P_{\psi}$ from the constant numerical value of the Hamiltonian $H=1+M^{2}$. This kind of launching condition initially places the system in the vicinity of the solitary solution, which is the solution containing the point $\Phi=P_{\Phi}=\psi=P_{\psi}=0$. In integrable cases the ensuing nearly solitary trajectories progress in fact as trains of solitons, but even in the nonintegrable cases where solitons are not seen, the trajectories still cross the $P_{\psi}=0$ plane several times, an essential condition for the construction of the maps.

We start by displaying in Fig. 4(a) the map obtained when $\Delta=0.98$. For such a value of $\Delta$ the adiabatic regime is expected to prevail. In agreement with that, what is seen in the plot is a set of regular orbital concentric curves. Note that the elliptic point appears to be located at a negative value of $\Phi$ simply because this is the value of the electric-field potential when the recording conditions $P_{\psi}=0, d P_{\psi} / d \xi>0$ are satisfied. Now if one starts to decrease $\Delta$ the transition to chaos is expected to occur. Let us move on to Fig. 4(b), where $\Delta=0.975$. As anticipated from the analytical estimates, a considerable amount of chaotic activity can already be identified. The central region of the map is completely surrounded by a blend of stochastic orbits and resonant islands. In particular, it appears that the soliton solution that corresponds to the central fixed point no longer exists. In Fig. 4(c) we enlarge part of Fig. 4(b) to show details of the resonant islands. These small remaining regions of regularity of the phase space are then totally suppressed when one reduces $\Delta$ further below. In Fig. 4(d), for instance, we consider the case $\Delta=0.97$ to show a deep chaotic regime. In conclusion, a complete destruction of the regular trapping region does indeed occur as $\Delta$ decreases. But if there is no trapping region, how would the trajectories behave? We will address this issue next. 

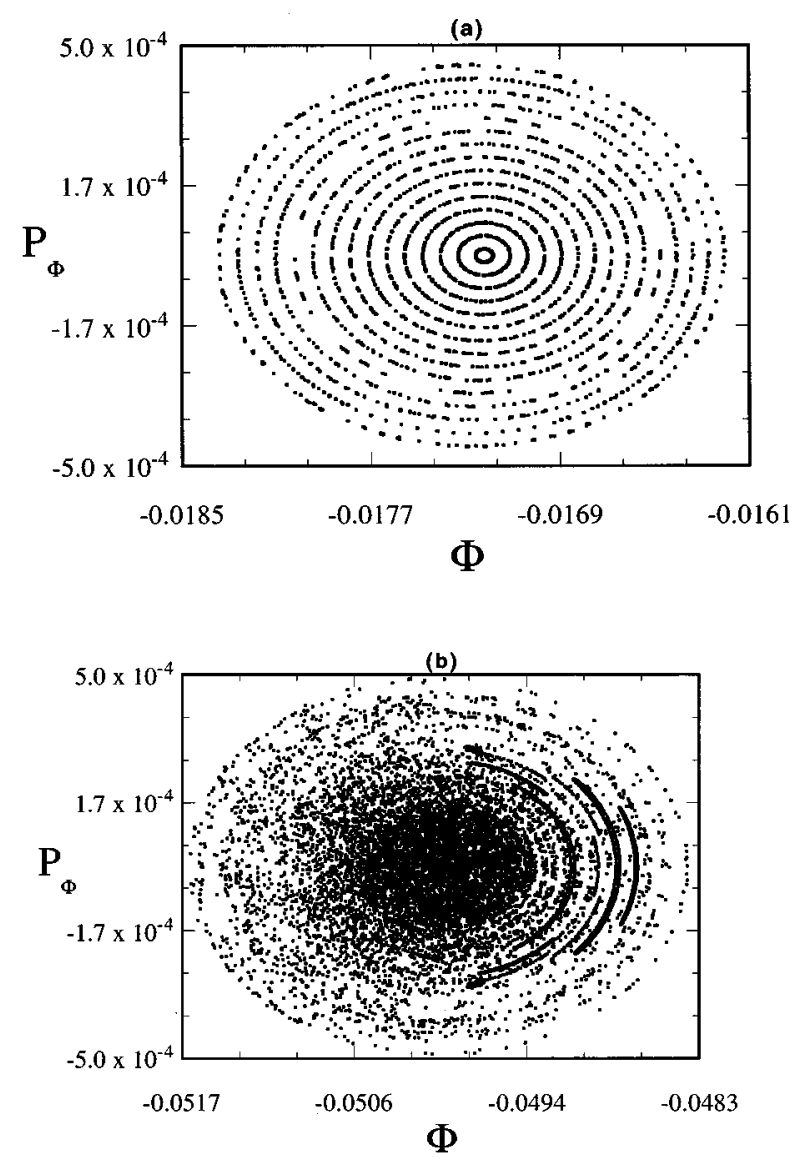

FIG. 4. Transition to chaos for $M=0.9$ and $\beta=100$ : (a) $\Delta=0.98$, (b) $\Delta=0.975$, and (d) $\Delta=0.97$. (c) Details of the resonant islands in
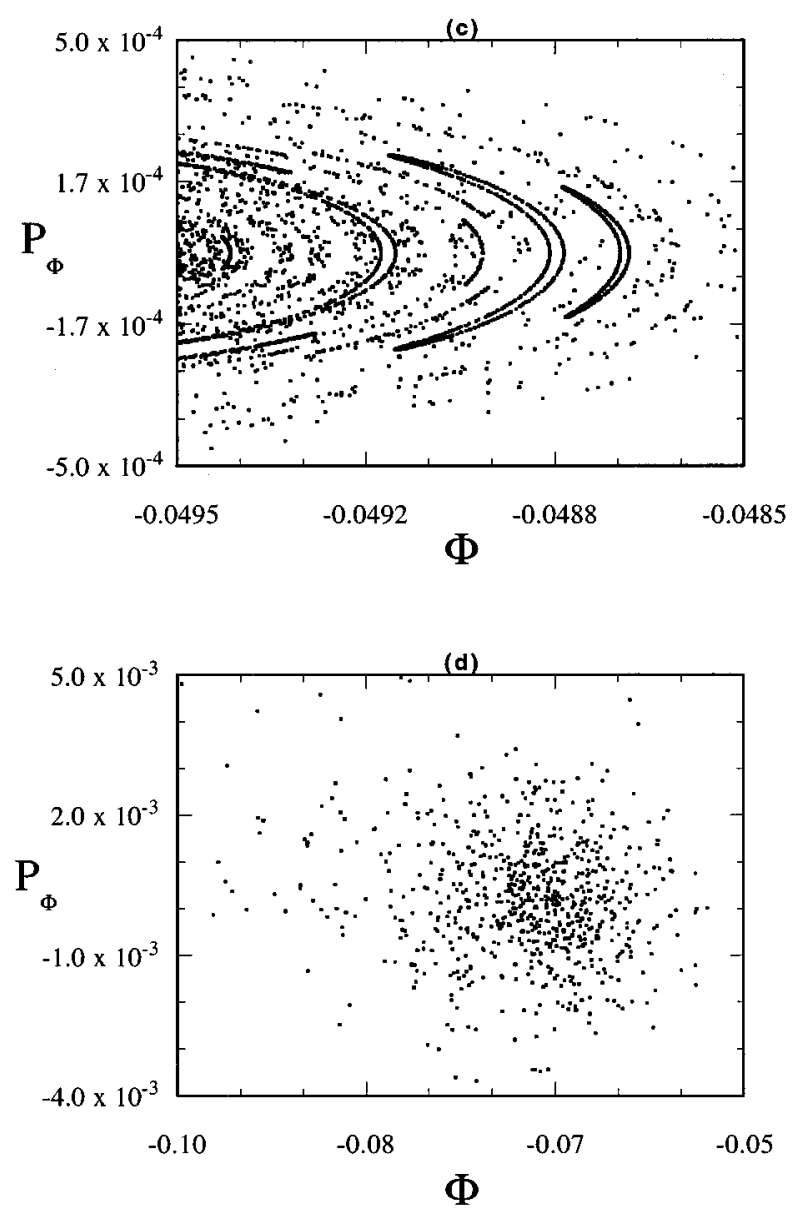
(b).

\section{Asymptotic states beyond the transition}

We can actually see some persistency in chaos only for those intermediary situations where $\Delta$ is not too close to unity, but not too small. Indeed, if $\Delta$ becomes sufficiently small, say $\Delta<\Delta_{\mathrm{cr}}$, no trapping region is effectively formed on the $\left(\Phi, P_{\Phi}\right)$ plane. Even initial conditions originally launched within the trapping region predicted by the adiabatic theory when $\psi=0$ do not remain there. As a matter of fact, the trajectories are eventually ejected into unbounded regions of the phase space when $\Delta$ crosses the validity limits of adiabaticity. For those cases, chaos would be at most a transient that would take place during initial instants, before ejection. The question to be asked now should be on the configuration of these unbounded orbits. What we have observed is that once the trajectory escapes from the trapping region in the ion acoustic phase space, it starts to follow the open flow lines of Fig. 1. This is confirmed in Fig. 5(a), where we show a continuous plot displaying a freed trajectory that was started with $\Delta=0.97$ and $\Phi(\xi=0)=0.0001$ [Figs. 5(a) and 5(b) are not Poincare maps; the trajectory points are periodically recorded with a small but constant time step]. The fact that the subsequent trajectory evolves along the flow lines implies that $\Phi$ gets more and more negative. Now, if one considers Eq. (8) one readily sees that regardless the value of $\psi$,

$$
\lim _{\Phi \rightarrow-\infty} H=\frac{P_{\psi}^{2}}{2 \beta^{2}}+\frac{\Delta \beta}{2} \psi^{2}-\frac{P_{\Phi}^{2}}{2}+M \sqrt{M^{2}-2 \Phi}
$$

In other words, laser and ion acoustic fields become decoupled in this limit. Since $\Delta \beta>0$, the corresponding dynamics of the laser field must necessarily become that of an undriven harmonic oscillator. This is what is shown in Fig. 5(b), where we project the same dynamics of Fig. 5(a), now on the $\left(\psi, P_{\psi}\right)$ phase plane. After a certain amount of time following a figure-eight shape like the ones seen in Figs. 1(c) and 3, there is a dynamical transition to the circular shape so characteristic of the harmonic oscillator. The instant of the transition coincides, as it should, with the moment of ejection seen in Fig. 5(a) and occurs approximately after 30 cycles of the laser wave in its initial figure-eight phase trajectory. Another interesting point connected to this asymptotic state is that as $\Phi \rightarrow-\infty$, the particle density becomes very small [see Eq. (7)]. Noticing that $\Phi<0$, the ponderomotive field created by the laser induces an initial potential well in which the interior of the ion fluid undergoes acceleration, thus becoming less dense. If the amplitude of the laser is too large the process is unstable and never arrests. When the density is low the laser becomes an almost standing wave with a small propagation velocity of the crests $(V \ll c)$. 

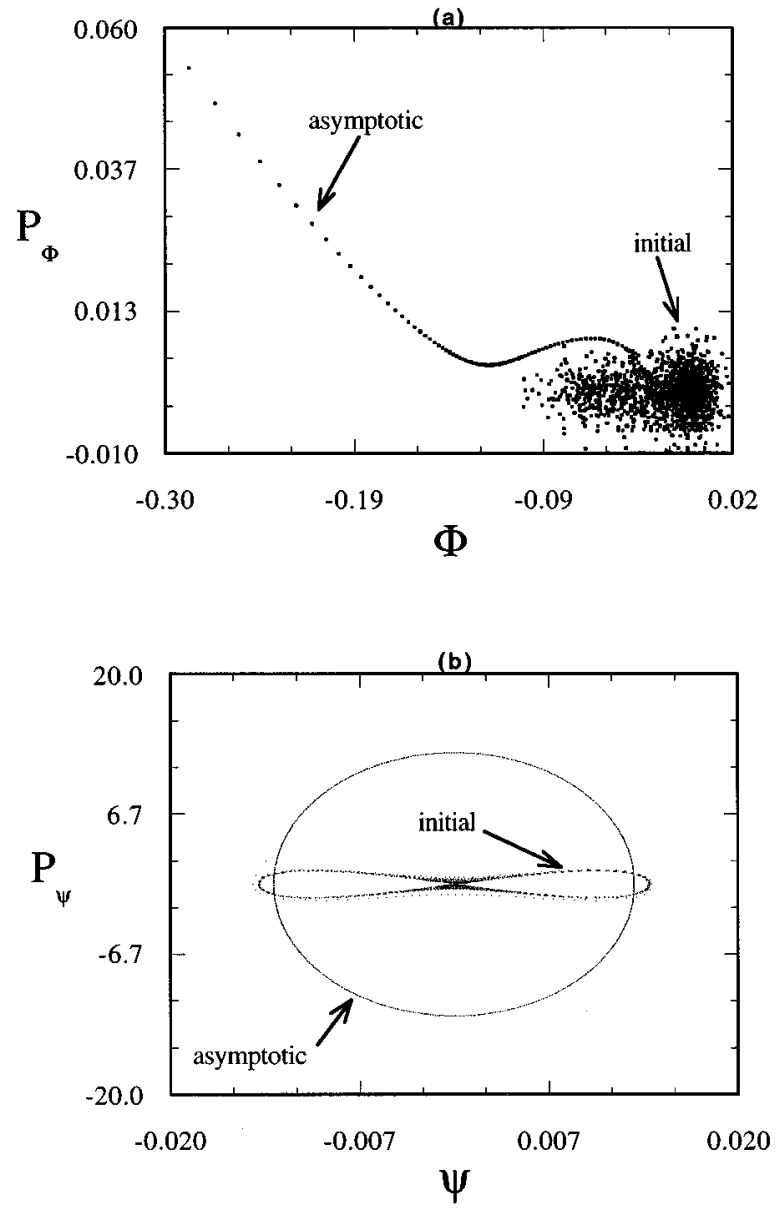

FIG. 5. Asymptotic states on the phase planes. (a) The escaping trajectory and (b) the circular trajectory appear on the respective phase planes after approximately ten figure-eight cycles of the $\psi, P_{\psi}$ variables: $M=0.9, \beta=100, \Delta=0.97$.

\section{Role of relativistic effects}

Our original equation (5) includes full relativistic electronic nonlinearities. As a final topic it is perhaps interesting to discuss the role of these relativistic nonlinear effects as compared to ponderomotive nonlinear effects.

What we find here is that while in the adiabatic regime, saturation is essentially governed by ponderomotive nonlinearities. This is the basic conclusion associated with Eq. (16). Now we would like to know whether or not relativistic effects grow in importance in chaotic regimes. To this end we perform two pairs of simulations, which are displayed in Fig. 6. In the upper panel of each pair we depict time series for $\Phi(\xi)$ considering exact fully relativistic nonlinear dynamics. In the lower panel we consider the time series with relativistic mass correction suppressed. It is seen that in the adiabatic regime of Fig. 6(a), where we consider $\Delta=0.98$, relativistic effects are not prominent, as both figures are almost identical. On the other hand, in Fig. 6(b), where we consider $\Delta=0.97$, relativistic effects are of relevance. Indeed, in the time series of Fig. 6(b) we see that for the particular initial conditions we use, the nonrelativistic trajectory undergoes much earlier ejection from the initial trapping region. For other initial conditions, the escape order may be
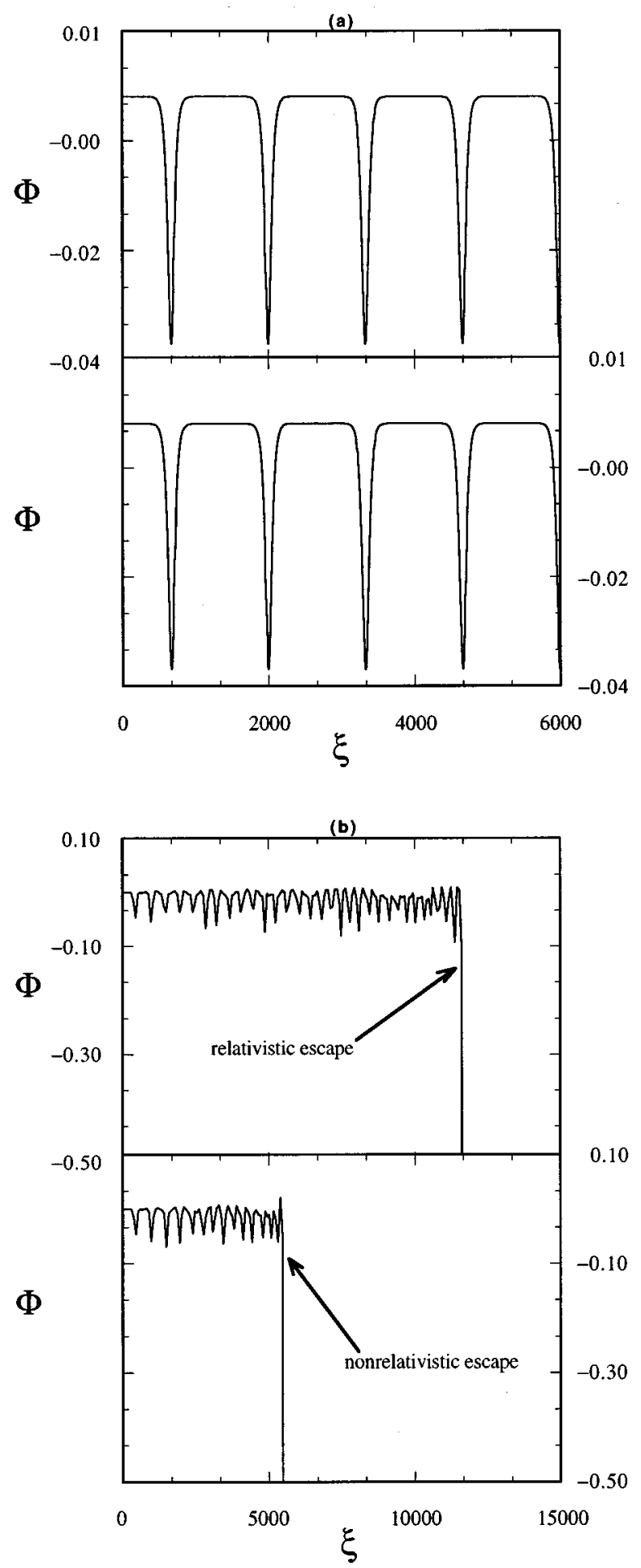

FIG. 6. Influence of relativistic nonlinearities on the dynamics. In the lower panel of each part the relativistic effect is artificially suppressed. (a) Adiabatic regular regime with $\Delta=0.98$ and (b) $\Delta=0.97$ is taken to produce an escaping trajectory after some transient chaos. It is seen that in the chaotic case one should not discard relativistic effects.

reversed and the nonrelativistic trajectory may be ejected after the relativistic one. What is really remarkable here, however, is that even in our case of small field amplitudes where relativistic effects are small, relativistic and nonrelativistic trajectories may largely differ in chaotic regimes, the reason 
for this being the extreme sensibility of chaotic systems to parameter variations.

\section{CONCLUSION}

We have performed a nonlinear analysis on the interaction of high-power laser waves with ion acoustic waves in a plasma. We assume stationary propagation in an overdense plasma and consequently show that three generic configurations take place. If the effective laser frequency is only slightly lower than the average electron plasma frequency, the ensuing dynamics is adiabatic. The faster varying ion acoustic field is adiabatically enslaved to the slowly varying envelope laser field and the resulting electromagnetic envelope solitons are likely to exist and remain stable. As one starts to decrease the effective laser frequency, adiabaticity becomes progressively poorer. A blend of confined chaotic regions and nonlinear resonance islands are seen on the $\left(\Phi, P_{\Phi}\right)$ phase space. Then, for yet smaller values of the laser frequency adiabaticity is completely destroyed. Initial conditions are rapidly ejected from the trapping region on the ion acoustic phase space and proceed to move along unbounded curves towards $\Phi \rightarrow-\infty$. In this limit the system becomes decoupled, the laser field starts to behave like a vacuum field, and the plasma becomes progressively rarefied.

Relativistic effects are moderate in adiabatic regimes, but of considerable relevance in chaotic regimes. We saw that trajectories with relativistic corrections artificially removed can largely differ from the exact ones, the reason being the sensibility of chaotic systems to parameter variations.

One general conclusion obtained here is that the parameter range for the existence of a solitary wave is extremely narrow. This numerically confirmed fact can be predicted only by nonlinear estimates such as that provided by Eq. (17), which takes into account the effect of finite values of $\psi^{2}$ on the existence of fixed points. Linear estimates such as that of Eq. (9) are much less accurate. Due to this narrow existence range, we have not observed, for instance, double hump solitons such as those obtained in Ref. [9]. The point is that according to the calculations done in this reference, and making the appropriate connections and translations between the various formulas, double hump solitons exist only when $\Delta$ reaches small values, $\Delta \sim 0.7$. However, for such a small value of this parameter, our system has already lost stability due to the transition to chaos.

\section{ACKNOWLEDGMENTS}

This work was partially supported by Financiadora de Estudos e Projetos (FINEP) and Conselho Nacional de Desenvolvimento Científico e Tecnológico (CNPq), Brazil. Numerical computing was performed on the CRAY Y-MP2E supercomputer at the Universidade Federal do Rio Grande do Sul Supercomputing Center.
[1] R.J. Noble, Phys. Rev. A 32, 460 (1985).

[2] W.L. Kruer, The Physics of Laser Plasma Interactions (Addison-Wesley, Reading, MA, 1988).

[3] J. Weiland and H. Wilhelmsson, Coherent Non-Linear Interaction of Waves in Plasmas (Pergamon, Oxford, 1977).

[4] A.C.-L. Chian, S.R. Lopes, and J.R. Abalde, Physica D (to be published).

[5] S.R. Lopes and A.C.-L. Chian, Phys. Rev. E 54, 170 (1996).

[6] A.C.-L. Chian and P.C. Clemmow, J. Plasma Phys. 14, 505 (1975).

[7] A.C.-L. Chian, Phys. Rev. A 24, 2773 (1981).

[8] P.K. Kaw, A. Sen, and E.J. Valeo, Physica D 9, 96 (1983).

[9] N.N. Rao, R.K. Varma, P.K. Shukla, and M.Y. Yu, Phys. Fluids 26, 2488 (1983).

[10] A.J. Lichtenberg and M.A. Lieberman, Regular and Stochastic Motion (Springer, Berlin, 1983).
[11] E. Infeld and G. Rowlands, Nonlinear Waves, Solitons and Chaos (Cambridge University Press, Cambridge, 1990).

[12] J.M. Wersinger, J.M. Finn, and E. Ott, Phys. Fluids 23, 1142 (1980).

[13] H.T. Moon, Phys. Rev. Lett. 64, 412 (1990).

[14] X.T. He and C.Y. Zheng, Phys. Rev. Lett. 74, 78 (1995).

[15] G.I. de Oliveira, L.P.L de Oliveira, and F.B. Rizzato, Phys. Rev. E 54, 3239 (1996); G.I. de Oliveira and F.B. Rizzato, Phys. Lett. A 214, 40 (1996); G.I. de Oliveira, F.B. Rizzato, and A.C.-L. Chian, Phys. Rev. E 52, 2025 (1995).

[16] J. Guckenheimer and P. Holmes, Nonlinear Oscillations, Dynamical Systems, and Bifurcations of Vector Fields (SpringerVerlag, New York, 1990).

[17] G. Corso and F.B. Rizzato, Physica D 80, 296 (1995); Phys. Rev. E 52, 3591 (1995). 